\title{
Les rhizotrons peuvent-ils être utilisés pour l'étude de la ramification des racines primaires nodales du maïs (Zea mays $L$ ) ?
}

\author{
MO Jordan 1 \\ avec la collaboration technique de C Vonarx 2 \\ 1 INRA, station d'agronomie, BP 91, 84143 Montfavet; \\ 2 INRA, station d'agronomie, BP 507, 68021 Colmar, France
}

(Reçu le 11 avril 1991; accepté le 25 novembre 1991)

\begin{abstract}
Résumé - Ce travail a pour objectif de discuter de la représentativité, par rapport aux conditions du champ, des mesures obtenues en rhizotrons, et concernant plus spécifiquement le nombre de secondaires sur les racines primaires du maïs (variété Dea).

Pour cela des échantillons ont été prélevés 2 années consécutives à la fois sur 6 parcelles, choisies de façon à permettre l'extériorisation de l'ensemble de la variabilité phénotypique, et en rhizotrons. Les dimensions des caissons sont les suivantes : profondeur $1 \mathrm{~m}$, largeur $20 \mathrm{~cm}$ et longueur $80 \mathrm{~cm}$. Le substrat utilisé est un sol reconstitué d'une des parcelles.

Nous avons montré que la variabilité des mesures sur le nombre de racines secondaires est importante et peut atteindre $30 \%$. En rhizotrons, les coefficients de variation sont encore plus élevés car viennent s'ajouter les conséquences des perturbations spécifiques à cette méthode.

Les observations effectuées in situ à travers la paroi transparente sont représentatives de ce qui se passe à l'intérieur du caisson. Cependant, ces résultats peuvent être transposés aux conditions du champ uniquement pour les entrenœuds 2 à 5 qui se ramifient en début de cycle. Ceci est vraisemblablement à relier au fait que les rhizotrons ont été mal isolés thermiquement. En effet, en fin de cycle, le refroidissement nocturne est particulièrement bien marqué en rhizotron.
\end{abstract}

Zea mays $\mathrm{L}=$ maïs / méthode / rhizotron / planche de fakir / développement racinaire / nombre de racines secondaires

Summary - Can rhizotrons be used for the study of corn (Zea mays L) root ramification? The aim of this work was to discuss the possibility to use rhizotrons to study the primary root ramification (number of organs) of corn plants (variety Dea). Samples, ie needle boards, have been taken 2 following years on 6 different fields chosen so that the whole genetic variability can be expressed. Measurements have also been made on rhizotrons of the following size: depth $1 \mathrm{~m}$, length $20 \mathrm{~cm}$, width $1 \mathrm{~m}$. The substrate is the soil of one of the fields. In 1985, one-third of the boxes were fitted out with a system (described in fig 2) for selecting the roots in contact with the transparent wall. Table I gives the number of evaluation performed for each method versus year and depth. The number of secondary roots per cm of primary roots versus internode and depth is given in figures 3 and 4 for the differents methods. The variability inherent in the ramification process is important and can attain $30 \%$. The coefficients of variation are greater for the results obtained with rhizotrons. They include the consequences of the specific perturbations due to the method. The observations made through the acrylic wall are representative of the ramification kinetics inside the box. Thus, figure 5 allows comparison of the different counts performed in the rhizotrons on the basis of the slope variation between 2 consecutive internodes. This figure shows that the acrylic wall did not disturb the secondary ramification, and that the results of the observations performed through the transparent face are proportional to those of the total number of ramifications on the observed primary roots. But these data can be extended to the field conditions for the first internodes $\left(E_{2}\right.$ to $E_{5}$ ) only. This is certainly caused by the soil temperature differences between the root boxes and the field which is especially important at the end of the vegetative growing season (fig 6) when the laterals on internode 6 and 7 are initiated. 


\section{INTRODUCTION}

Les rhizotrons, décrits pour la premiere fois par Taylor (1969), sont maintenant couramment utilisés pour l'étude du système racinaire. D'un coût relativement modeste, ils permettent de réduire considérablement la pénibilité et la durée d'acquisition des données expérimentales (Huck et Taylor, 1982).

Cependant cette technique introduit des biais spécifiques qui rendent difficile la transposition des résultats aux conditions du champ, niveau d'étude qui intéresse plus particulièrement l'agronome.

Le comportement de l'ensemble du végétal : rapport des biomasses aériennes et souterraines, longueur et densité racinaire par horizon de sol est souvent modifié du fait que l'on travaille sur une plante isolée (Raper et Barber, 1970 ) et cultivée en conteneurs. Celui-ci limite le volume de sol disponible pour la plante (Peterson et al, 1984), et implique l'utilisation d'un milieu de culture (sol ou substrat du type argile expansée) dont la structure est perturtée, et dont la température est généralement différente de celle du sol.

Les racines observées sont celles au contact d'une paroi transparente, souvent électrostatique et dont l'adhérence avec le substrat n'est pas parfaite. L'effet induit sur la densité racinaire est fonction de la nature de cette paroi (verre ou plexiglass) et de l'espèce végétale (Taylor et Bohm, 1976; Voorhees, 1976; Bohm, 1979).

L'influence de ces facteurs sur l'enracinement a été quantifiée uniquement pour les densités:

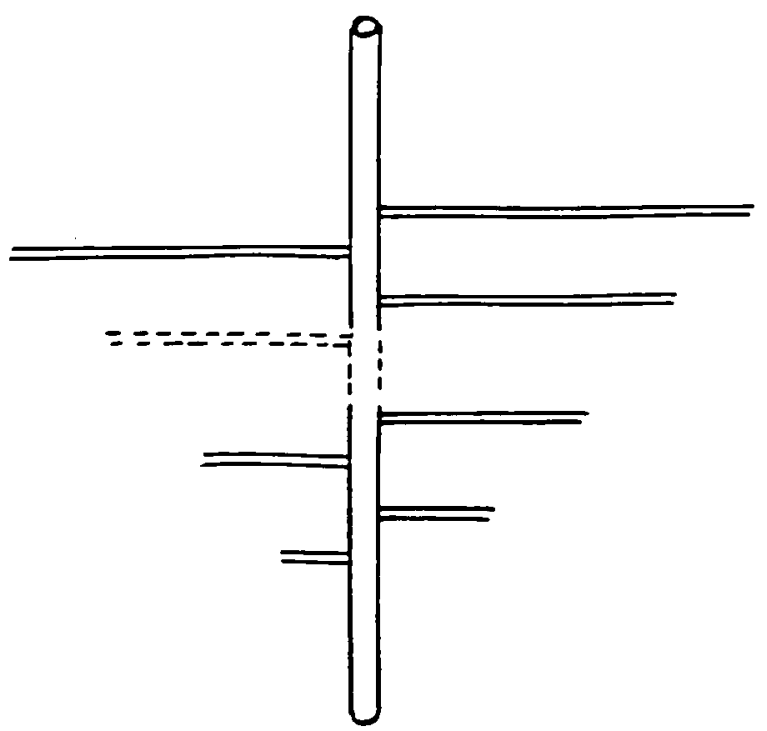

longueur de racines par $\mathrm{cm}^{3}$ de sol (Taylor et Bohm, 1976; Voorhees, 1976). Nous nous proposons ici d'en évaluer les conséquences sur l'organogenèse, et plus particulièrement sur les nombres de racines secondaires (terminologie définie par Girardin et al, 1986). En effet, c'est la nécessité d'accéder à ce type d'informations, caractéristique de la structure racinaire et difficile à obtenir au champ (obligation de prélever des portions de primaires relativement longues dont on connaît l'entre-nœud d'insertion sur la tige) qui justifie l'emploi des rhizotrons.

Notre étude portera sur maïs (variété Dea). Après avoir présenté les résultats de nos observations effectuées au travers de la paroi transparente, nous discuterons leur validité par rapport à des mesures effectuées à l'intérieur du caisson d'une part, et au champ d'autre part.

\section{DISPOSITIF EXPÉRIMENTAL}

\section{Ramification d'une racine primaire}

Chaque racine est composée de 3 parties distinctes (fig 1) : 2 zones non ramifiées $a$ et $b$ situées respectivement en-deça de l'apex et à la base, sous le point de raccordement à la tige, entourent la partie centrale $r$, d'où sont issues les ramifications secondaires.

Les longueurs de $a$ et $b$ sont fonction de l'entre-nœud d'insertion de la racine primaire génératrice du réseau. Elles ne varient pas au cours du cycle de culture. Une racine commence donc à se ramifier dès que sa longueur est égale

$$
\begin{aligned}
\text { b : partie non ramifiée située } \\
\text { a la base de la rocine sous } \\
\text { son point de raccordement }
\end{aligned}
$$

\section{$r$ : zone ramifié dont la longueur} s'accroit pendant la croissance
a : partie non ramifiée situee en-deça de l'extrémité apicale

Fig 1. Différentes parties d'une racine. 
à $(a+b)$ et le front d'émission racinaire va progresser le long de la primaire au fur et à mesure de son élongation. Nous n'avons jamais observé de ré-émission sur un tronçon déjà ramifié : le nombre total de racines secondaires s'y stabilise quelques jours après le début de la ramification (Jordan et al, 1992). Nous discuterons ici des mesures effectuées sur la partie $r$ (nombre de secondaires par $\mathrm{cm}$ de primaire) à partir de la date de fin d'émission.

\section{Le dispositif}

Les résultats obtenus en rhizotrons ont été évalués par rapport à ceux du champ de 1983 à 1985. Le dispositif utilisé à déjà été décrit de façon précise dans Jordan (1986). Seuls les points les plus importants seront repris ici.

\section{Les rhizotrons}

Six rhizotrons ont été utilisés en 1984. Ce nombre a été doublé en 1985 en raison de l'utilisation d'une plaque de sélection des primaires, décrite ultérieurement, qui limite le nombre d'observations possibles.

La dimension des rhizotrons est telle que leur section est analogue à la surface au sol dont dispose une plante au champ $(80 \times 20 \mathrm{~cm})$. La profondeur est de $1 \mathrm{~m}$, Grimes et al (1975) ayant montré qu'en conditions irriguées, $99 \%$ de la matière sèche racinaire était située dans les 90 premiers $\mathrm{cm}$ de sol.

Le caisson est en PVC et isolé thermiquement à l'aide de polystyrène expansé. L'ensemble du rhizotron est incliné de $15^{\circ}$ du côté de la paroi transparente en plexiglas. Entre 2 mesures, celleci est recouverte d'un cache de PVC opaque.

Des tensiomètres, placés respectivement à 25 , 50 et $75 \mathrm{~cm}$ de profondeur, permettent de vérifier que l'alimentation hydrique est non limitante. $\mathrm{Par}$ ailleurs, $1 / 3$ des caissons ont été équipés aux mêmes profondeurs de sondes thermiques (soil moisture equipment, cat 5500 - Santa-Barbara). Les relevés sont manuels et effectués journellement à $9 \mathrm{~h}$ du matin afin d'homogénéiser nos données avec celles de la station météo située sur le centre.

En 1984, nous avons été gênés dans nos comptages par des chevauchements racinaires consécutifs au trop grand nombre d'organes au contact de la paroi transparente. En 1985, 8 rhizotrons ont donc été équipés d'un plaque de
PVC (schématisée fig 2) qui écartait le système racinaire de la vitre. Seules 4 racines, choisies en fonction de leur entre-nœud d'insertion, sont amenées au contact de celle-ci : pour cela, elles sont passées au travers de trous percés dans la plaque, trous prolongés par une gaine de PVC qui les relie à la paroi. Pour faciliter les manipulations, le substrat décrit ultérieurement a été remplacé au-dessus de ce dispositif par des billes d'argile expansée de diamètres compris entre 4 et $8 \mathrm{~mm}$. Pendant la phase d'implantation des plantules, cette juxtaposition de 2 milieux a provoqué un arrêt de croissance qui n'a pas eu d'effet sur la production de biomasse totale.

Les caissons sont placés sous abri mobile et remplis avec un sol remanié dont les caractéristiques physico-chimiques sont celles de la parcelle de Colmar 1 (Jordan, 1986). Le profil du sol a été reconstitué avec ses différents horizons. Une couche drainante d'une épaisseur de $10 \mathrm{~cm}$, composée de gravier et de sable, occupe le fond des rhizotrons.

Les semis, de 4 plantes par caisson, sont effectués le 9 mai en 1984 et le 24 avril en 1985. Au stade 3 feuilles, un seul pied, choisi de façon à ce que la population soit la plus homogène possible, est conservé. Le décalage des dates, de semis se retrouve à la floraison femelle qui a lieu le 10 août en 1984 et le 30 juillet en 1985. La production de biomasse aérienne a été supérieure de $42 \%$ à celle du champ : ceci est certainement dû au fait que l'on travaille sur des végétaux isolés disposant d'un volume de sol suffisant.

\section{Le champ}

L'expérimentation a été réalisée en 1983 et 1984 sur 6 parcelles conduites selon les pratiques de la grande culture.

Les sites ont été choisis de façon à inclure une certaine fluctuation climatique, notamment du point de vue de la pluviométrie totale qui, pour les mois d'avril à octobre, varie de 347 à $623 \mathrm{~mm}$ entre les différentes localisations.

Les sols des stations sont formés sur loess et présentent tous une texture de limon argileux avec une teneur en matière organique faible à modérée, un $\mathrm{pH}$ alcalin ou légèrement acide.

Les techniques culturales ont été laissées à l'initiative des agriculteurs.

Les semis, d'une densité comprise entre 7,4 et 9,5 pieds $/ \mathrm{m}^{2}$ ont été échelonnés du 24 avril au 29 avril en 1983 et du 23 avril au 3 mai en 1984 , les floraisons femelles du 18 juillet au 25 


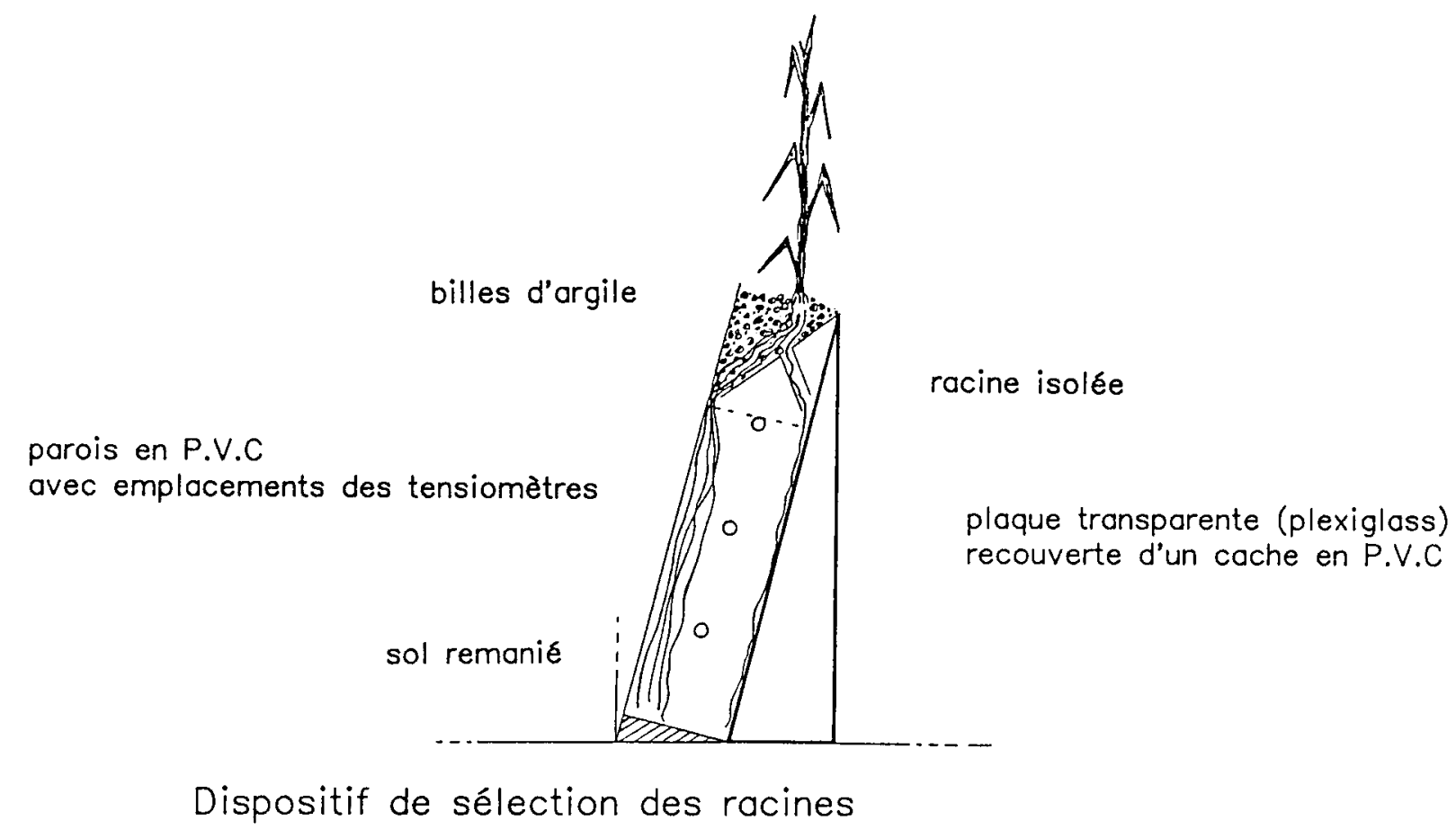

Vue de face

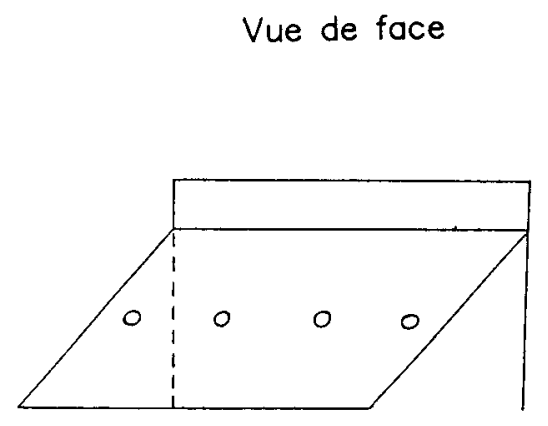

Vue de profil

Fig 2. Schéma d'un rhizotron avec le dispositif de sélection des racines.

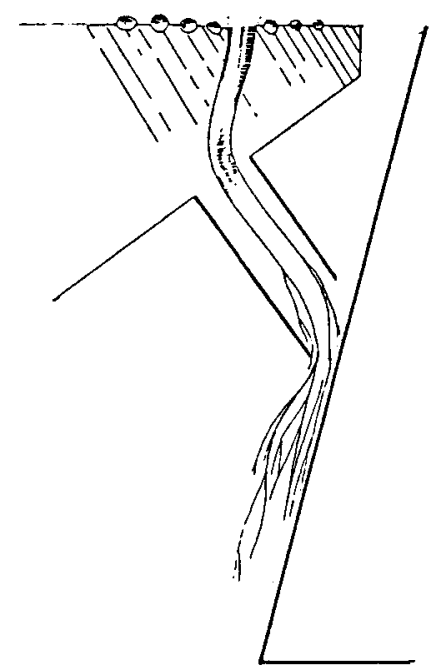

juillet en 1983 et du 30 juillet au 8 août en 1984 . Les rendements en grains d'une parcelle donnée ne varient pas significativement d'une année à l'autre et sont compris entre 78 et $103 \mathrm{q} / \mathrm{ha}$.

\section{Les mesures}

\section{Les rhizotrons}

Chaque primaire au contact de la vitre est individualisée et le numéro de l'entre-nœud dont elle est issue déduit du nombre de feuilles totales (d'après les résultats de Picard et al, 1985). Après ramification, le nombre de racines d'ordre 2 est évalué. Les comptages sont faits par tronçon de $5 \mathrm{~cm}$ à partir de la base, sur la partie visible de la racine porteuse.

À maturité, la paroi transparente est ôtée et les primaires, au contact de celle-ci, prélevées une à une. Les mêmes déterminations sont alors effectuées sur toute la circonférence de la racine. L'emploi de la binoculaire s'impose en raison du nombre important de ramifications et de la nécessité de comptabiliser les moignons des organes brisés au cours des manipulations. 
Enfin, le système racinaire restant, pris dans la masse de terre, est dégagé par aspersion, et les mêmes comptages sont réalisés sur chaque primaire.

\section{Le champ}

Les observations ont été effectuées à partir de planches de fakir d'une épaisseur de $15 \mathrm{~cm}$, d'une largeur de $82 \mathrm{~cm}$ et d'une profondeur de $42 \mathrm{~cm}$. Pour les 6 sites, 2 prélèvements perpendiculaires au rang de maïs ont eu lieu à la floraison selon un procédé décrit par De Roo (1957), Nelson et Allmaras (1969); les plantes échantillonnés se trouvant au centre de la section $82 \mathrm{x}$ $15 \mathrm{~cm}$ du monolithe.

Les mesures, entreprises après élimination de la terre à l'aide d'un jet d'eau à faible pression, sont celles décrites précédemment.

\section{RÉSULTATS}

Le nombre de ramifications par unité de longueur est fonction de l'année et de 2 paramètres caractérisant la primaire porteuse : le rang de son entre-nœud d'implantation (E) sur la tige et la distance entre ce point d'insertion et la zone étudiée. Concernant ce dernier point, la discrimination en classes $(0-35,35-60 \mathrm{~cm}$ et $60-90 \mathrm{~cm}$ ) correspondant grossièrement aux portions de racines colonisant chacun des horizons de sol est suffisante (Jordan et al, 1992).

Les résultats obtenus seront donc exprimés en fonction de ces 3 facteurs. Les comptages ayant eu lieu sur toutes les primaires existantes, le nombre de répétitions (tableau I) varie fortement selon les cas.

\section{Les rhizotrons}

Les résultats sont présentés figures 3 et 4 . Aucune donnée pour l'horizon $0-35 \mathrm{~cm}$ n'a pu être obtenue en 1985, en raison de la méthode employée pour dévier les racines vers le fond du rhizotron.

\section{Observations à travers le plexiglas}

Les racines d'ordre 2 visibles in situ, sont celles émises sur le quart, voire le tiers de la circonférence de la primaire. Leur nombre, par unité de longueur de $1 \mathrm{~cm}$, varie, selon les entre-nœuds de 1,2 à 1,9 en 1984 et de 1,3 à 2,5 en 1985. La valeur des coefficients de variations (CV) sur ces moyennes oscille respectivement de 39 à $58 \%$ en 1984 et de 46 à $56 \%$ en 1985 . Ce manque de précision sur les résultats est vraisemblablement à relier au fait que le nombre de secondaires visibles est fonction de la qualité du contact entre la terre et la paroi. Il est plus élevé si l'adhérence est mauvaise, par exemple, s'il existe des fentes de retraits.

En 1985, le dispositif de sélection limite la densité racinaire à l'interface sol-plexiglas; les chevauchements sont réduits mais le nombre de déterminations plus limité. Ceci explique en partie, l'augmentation observée en 1985, d'une part sur les CV (baisse de précision sur les mesures), et d'autre part sur les valeurs moyennes (visibilité de comptage bien meilleure). Ces différences interannuelles sont cependant non significatives statistiquement (tests de Bonferroni, seuil $5 \%)$.

\section{Observations à la destruction des rhizotrons}

Au contact de la paroi transparente, le nombre total de secondaires, c'est-à-dire celles émises sur toute la circonférence des primaires, varie, selon les entre-nœuds, de 2,7 à 7,4 (CV moyen de $36 \%$ ) en 1984 et de 6,3 à 9,2 (CV moyen de $42 \%$ ) en 1985 . Les racines d'ordre 1 qui se sont développées au cœur du monolithe de terre portent de 3,4 à 10,5 ramifications par $\mathrm{cm}$ en 1984 (CV moyen $32 \%$ ) alors qu'en 1985, cet intervalle est compris entre 3,4 et 8,8 (CV moyen de $34 \%)$.

Des tests de comparaison de moyennes (Bonferroni, seuil 5\%) montrent :

- que les taux de ramification des primaires au cœur du monolithe de terre sont comparables les 2 années d'étude; notons cependant que les différences entre les moyennes peuvent être masquées par l'importance des écarts types;

- que le plexiglas a un effet dépressif sur l'émission des racines secondaires, mais uniquement en 1984. Nous ne disposons d'aucun indice permettant de définir la raison de ce comportement : variations interannuelles des conditions pédo-climatiques ou influence du dispositif de sélection.

\section{Comparaison des différents comptages}

Notons tout d'abord que la précision des résultats est fonction de la méthode utilisée. Les CV 
Tableau I. Nombre de répétitions pour chaque type de comptage.

\begin{tabular}{|c|c|c|c|c|c|c|c|}
\hline Méthode & Horizon & $\mathrm{E}_{2}$ & $E_{3}$ & $E_{4}$ & $\mathrm{E}_{5}$ & $\mathrm{E}_{6}$ & $E_{7}$ \\
\hline \multicolumn{8}{|l|}{ Rhizotrons 1984} \\
\hline Vitre en place & $\begin{array}{l}0 / 35 \mathrm{~cm} \\
35 / 60 \mathrm{~cm} \\
60 / 90 \mathrm{~cm}\end{array}$ & $\begin{array}{l}216 \\
152 \\
130\end{array}$ & $\begin{array}{r}141 \\
80 \\
72\end{array}$ & $\begin{array}{r}103 \\
80 \\
65\end{array}$ & $\begin{array}{l}65 \\
73 \\
45\end{array}$ & $\begin{array}{l}97 \\
57 \\
17\end{array}$ & $\begin{array}{r}100 \\
27 \\
10\end{array}$ \\
\hline Vitre : récolte & $\begin{array}{l}0 / 35 \mathrm{~cm} \\
35 / 60 \mathrm{~cm} \\
60 / 90 \mathrm{~cm}\end{array}$ & $\begin{array}{l}61 \\
42 \\
35\end{array}$ & $\begin{array}{l}46 \\
31 \\
15\end{array}$ & $\begin{array}{l}39 \\
35 \\
35\end{array}$ & $\begin{array}{l}54 \\
49 \\
45\end{array}$ & $\begin{array}{l}79 \\
63 \\
52\end{array}$ & $\begin{array}{r}168 \\
98 \\
64\end{array}$ \\
\hline Terre : récolte & $\begin{array}{r}0 / 35 \mathrm{~cm} \\
35 / 60 \mathrm{~cm} \\
60 / 90 \mathrm{~cm}\end{array}$ & $\begin{array}{l}66 \\
40 \\
29\end{array}$ & $\begin{array}{l}54 \\
45 \\
45\end{array}$ & $\begin{array}{l}48 \\
32 \\
30\end{array}$ & $\begin{array}{l}36 \\
36 \\
30\end{array}$ & $\begin{array}{l}51 \\
37 \\
33\end{array}$ & $\begin{array}{r}116 \\
77 \\
70\end{array}$ \\
\hline
\end{tabular}

Rhizotrons 1985

Vitre en place

Vitre : récolte

Terre : récolte

Planches de fakir 1983
$35 / 60 \mathrm{~cm}$ $60 / 90 \mathrm{~cm}$

$0 / 35 \mathrm{~cm}$

$35 / 60 \mathrm{~cm}$

$60 / 90 \mathrm{~cm}$

$0 / 35 \mathrm{~cm}$

$35 / 60 \mathrm{~cm}$

$60 / 90 \mathrm{~cm}$

$0 / 35 \mathrm{~cm}$

109

101

163

149

177

147

Planches de fakir 1984

$0 / 35 \mathrm{~cm}$

93

117

108

125

109

74

les plus faibles sont ceux relatifs aux données obtenues sur les primaires au cœur du monolithe, non soumises aux perturbations liées à la présence du plexiglas.

Le tableau II donne les résultats d'une analyse de variance en modèle linéaire général sur les données obtenues en rhizotrons. Chacun des facteurs considérés, est significatif avec une probabilité de 1/1 000. L'effet année s'explique par les différences observées à maturité sur les primaires au contact de la vitre (cf paragraphe précédent). Les influences de l'entre-nœud d'insertion $(E)$ et de l'horizon de sol sont inhérentes au processus de ramification (Jordan et al, 1991). Cependant, elles ne concernent que les valeurs moyennes. L'analyse des interactions montre en effet, que, pour chaque type de comptage, les cinétiques d'évolution du nombre de secondaires en fonction de $E$ (figs 4 et 5 ) sont comparables pour les 2 années et les 3 horizons pris en compte.

II est plus délicat d'évaluer la représentativité des résultats obtenus in situ sur les primaires au contact du plexiglas : les interactions concernant la technique de comptage sont en effet significatives (tableau II). II est donc nécessaire de procéder à des examens complémentaires. L'absence d'une relation de proportionnalité indépendante de $E$ (rang d'insertion de la primaire sur la tige) entre les résultats obtenus in situ, d'une part, et à la destruction des rhizotrons, d'autre part, nous oblige à nous intéresser à l'allure générale des courbes décrites figures 3 et 4 . Mais, les intervalles de confiance sont trop larges pour qu'il soit possible de nous limiter à la comparaison des modèles décrivant l'évolution de ces 


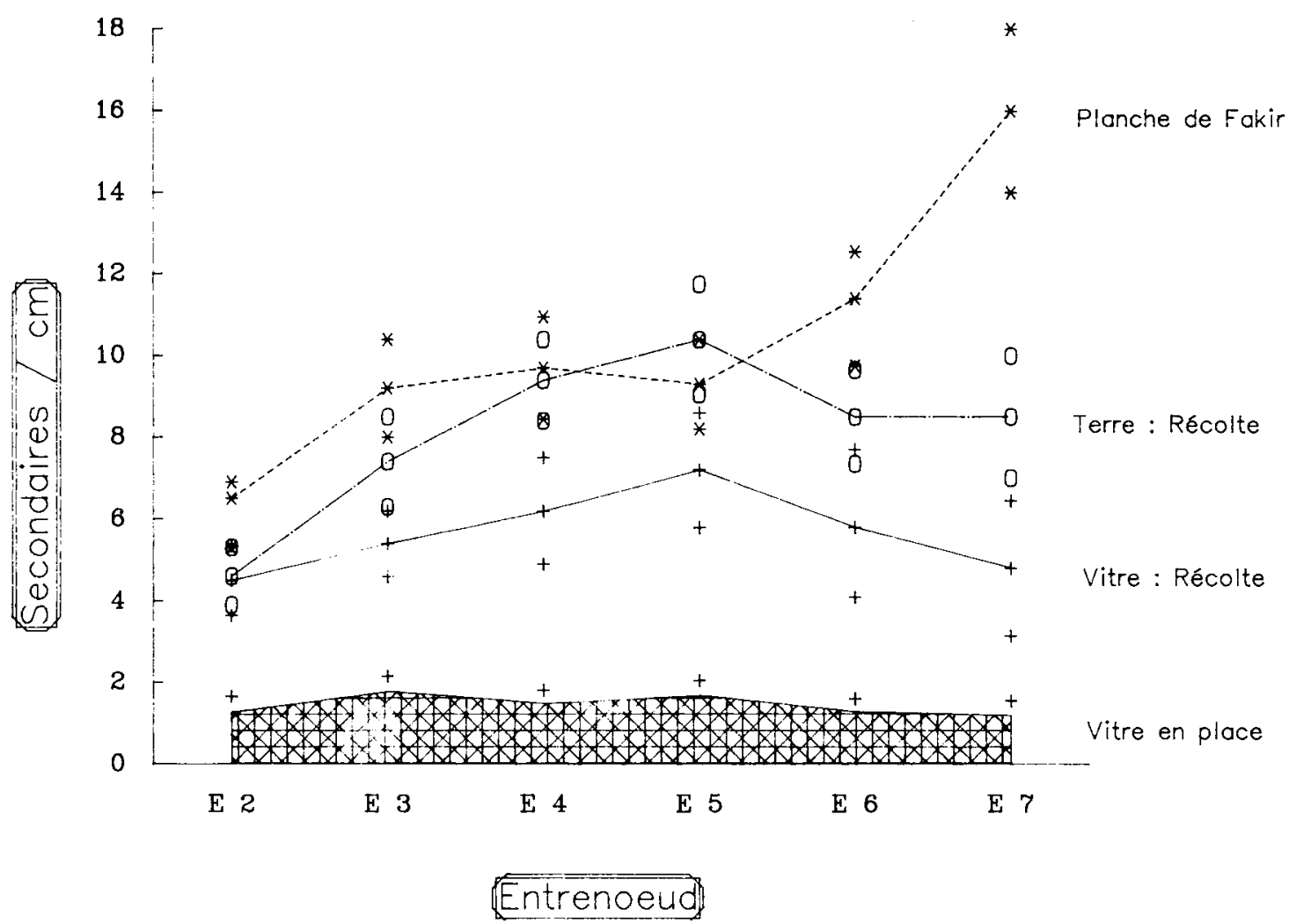

Fig 3. Pour chacune des méthodes employées, nombre de racines secondaires par $\mathrm{cm}$ en fonction du rang d'insertion de la racine primaire. Résultats obtenus en 1984, pour l'horizon $0 / 35 \mathrm{~cm}$.

Tableau II. Résultats des analyses de variance.

Facteur Degrés de liberté F

\section{Rhizotrons}

$\begin{array}{lrc}\text { Année } & 1 & 42,69^{\star \star \star} \\ \text { Méthode } & 2 & 341,32^{\star \star \star} \\ \text { Horizon } & 2 & 21,41^{\star \star \star} \\ \text { Entre-nœud } & 5 & 14,01^{\star \star \star} \\ \text { Année-méthode } & 2 & 21,80^{\star \star \star} \\ \text { Année-horizon } & 1 & 3,59 \\ \text { Année-entre-nœud } & 5 & 2,65 \\ \text { Méthode-horizon } & 4 & 5,54^{\star \star \star} \\ \text { Méthode-entre-nœud } & 10 & 3,47^{\star} \\ \text { Horizon-entre-nœud } & 10 & 1,13 \\ \text { Résiduelle } & 45 & \end{array}$

\section{Planches de fakir}

$\begin{array}{lrc}\text { Site } & 6 & 1,59 \\ \text { Année } & 1 & 5,69^{\star} \\ \text { Entre-nœud } & 5 & 20,53^{\star \star *} \\ \text { Résiduelle } & 59 & \end{array}$

* : résultat significatif au seuil de $1 \%$; "** : résultat significatif au seuil de $5 \%$. courbes. Nous avons donc travaillé (fig 5) sur les valeurs moyennes $(P-x)$ et les écarts types $(P-E T)$ des pentes entre 2 points (ou entrenœuds) successifs. Les valeurs moyennes ( $P-$ $x$ ), comprises entre 0,5 et 2,5 sont du même ordre de grandeur quelle que soit la méthode utilisée. Leurs écarts types $(P-E T)$, proportionnels à la variance des nombres moyens de secondaires par entre-nœud, sont plus importants sur les déterminations réalisées à maturité que sur les mesures in situ.

Les tirets, figure 5 , symbolisent les intervalles de confiance $(P-E T)$ sur les pentes des courbes relatives au taux de ramification des organes qui se sont développés au cœur du monolithe de terre. Les moyennes $P-x$ concernant les 2 autres méthodes de comptage, et plus particulièrement les observations in situ (symbolisées par un trait plein figure 5) se situent, le plus souvent, entre les bornes de la référence choisie. Les mesures effectuées à l'interface sol-paroi donnent donc une image relativement juste de ce qui se passe à l'intérieur du rhizotron, sauf pour $E_{2} / E_{3}$. L'adéquation est meilleure en 1985 , où la densité racinaire a été limitée au contact du plexiglas. 


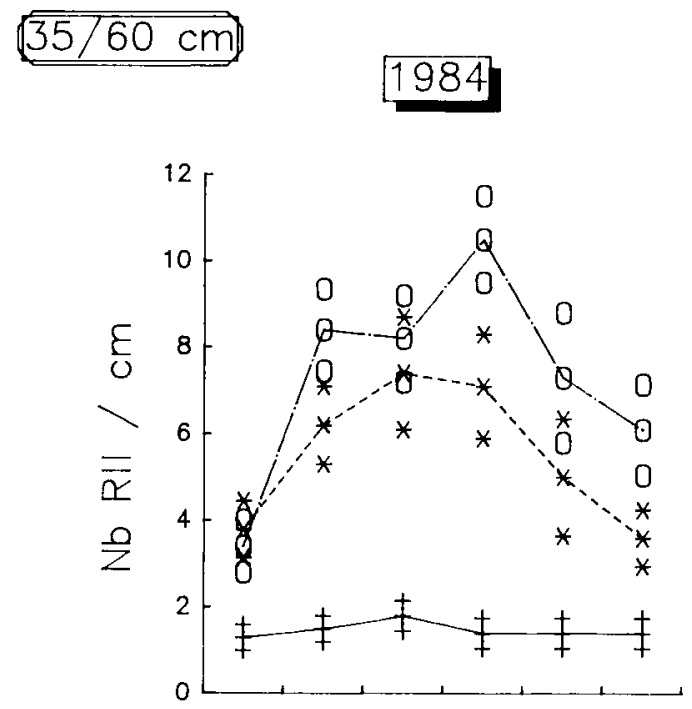

$60 / 90 \mathrm{~cm}$
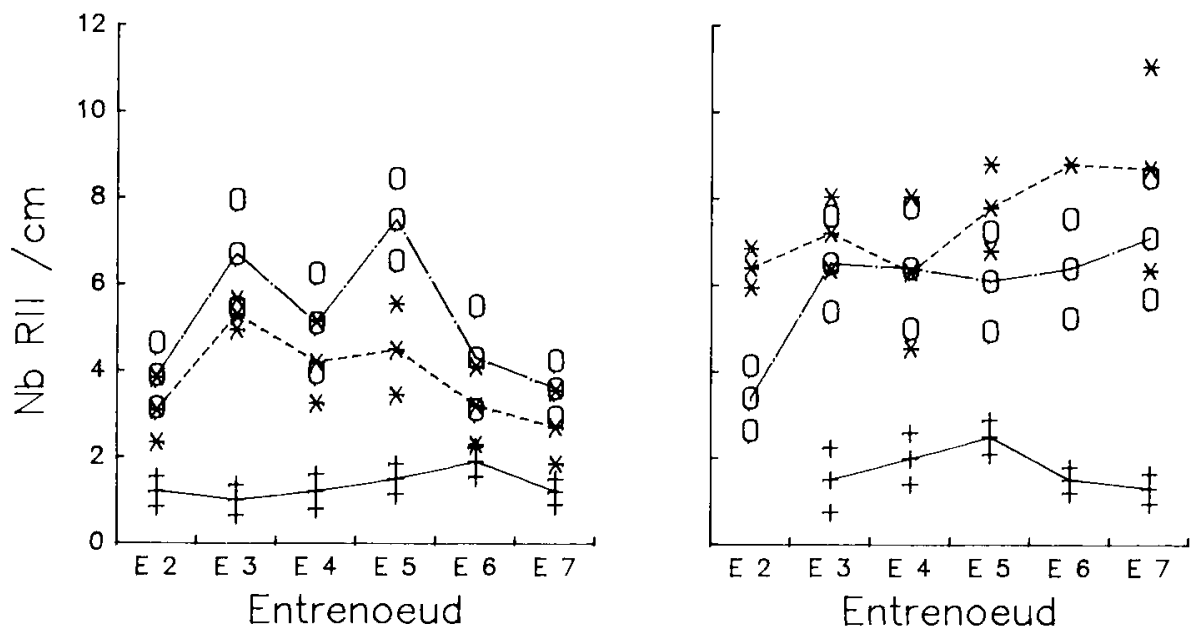

Légende : 0 : Terre, Récolte /*: Vitre, Récolte $/+$ : Vitre en place

Fig 4. Pour chacune des méthodes employées, nombre de racines secondaires par $\mathrm{cm}$ en fonction du rang d'insertion de la racine primaire. Résultats 1984 et 1985 obtenus en rhizotrons, pour les horizons $35 / 60 \mathrm{~cm}$ et $60 / 90 \mathrm{~cm}$.

\section{Les planches de fakir}

\section{Résultats bruts}

La figure 3 regroupe, pour l'année 1984, les résultats des 6 localisations, une analyse de variance (tableau II) ayant montré que ce facteur n'est pas significatif.

Le nombre de ramifications par $\mathrm{cm}$ augmente avec le rang de l'entre-nœud (seuil $1 / 1000$ ) et passe de 6,5 pour $E_{2}$ à 13,1 pour $E_{7}$. Les va- leurs obtenues en 1984, comprises entre 6,1 et 16,0 sont significativement plus élevées (seuil 1/ 100 ) sauf pour $E_{2}$; l'allure générale de la courbe n'étant pas modifiée.

Pour l'ensemble des mesures les CV sont constants et relativement faibles (environ $28 \%$ ).

\section{Adéquation avec les données rhizotrons}

Une comparaison de ces données avec les nombres de secondaires portées par les primaires qui se sont développées au cœur du mo- 


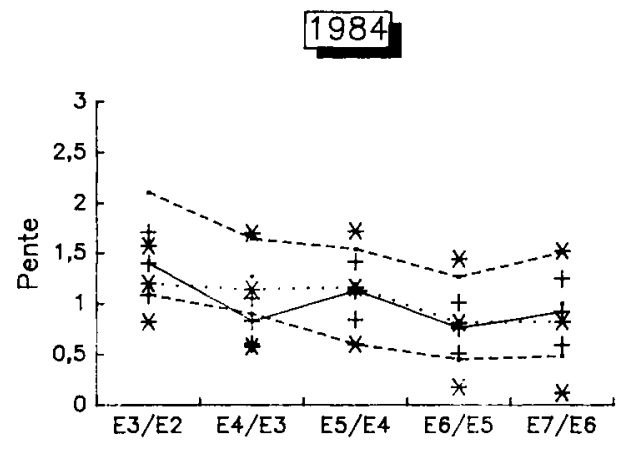

\section{Légende :}

., tirets : Terre, Récolte

*, pointillés : Vitre, Recolte

+ , traits pleins : Vitre en ploce

\section{$3 5 \longdiv { 6 0 \mathrm { cm } }$}
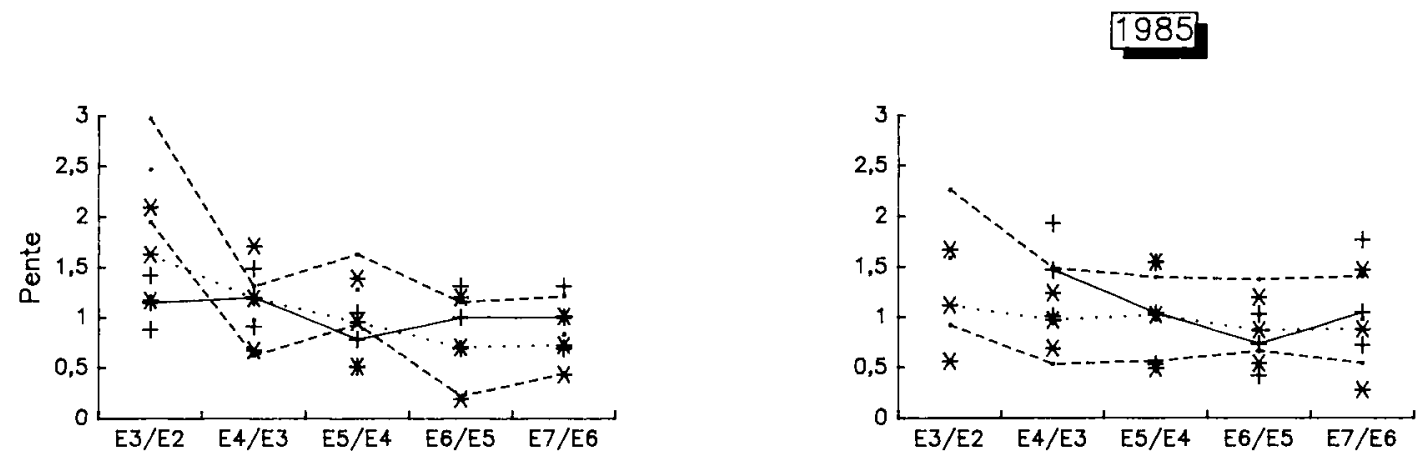

$6 0 \longdiv { 9 0 \mathrm { cm } }$
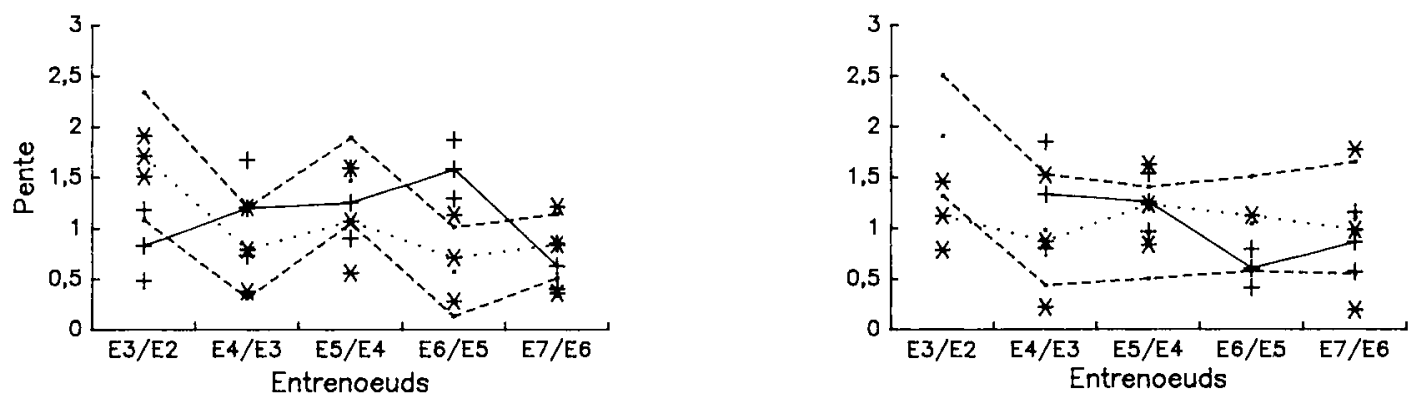

Fig 5. Comparaison des résultats obtenus en rhizotrons à l'aide des différentes méthodes.

nolithe de terre (milieu le plus proche des conditions du champ) montre que :

- la précision sur les mesures est sensiblement la même au champ et en caisson : les différences sur les CV sont inférieures à $5 \%$ donc peu significatives;

- les taux de ramification (fig 3) sont moins importants en rhizotrons. De plus, les évolutions du nombre de ramifications en fonction du rang de l'entre-nœud ne sont pas comparables : elles semblent être spécifiques du mode de culture et indépendantes de l'année. Au champ, les courbes (fig 3, pour 1984) sont ascendantes sur l'ensemble du cycle alors qu'en container, on observe une baisse marquée pour $E_{6}$ et $E_{7}$ (figs 3 et 4). 
Ces différences peuvent s'expliquer, en partie, par le fait que la température du sol est modifiée en rhizotron. Les caissons n'étant pas enterrés, les valeurs mesurées sont les mêmes quelle que soit la profondeur et présentent des variations journalières importantes en relation avec celles de l'air. De même, le rythme nycthéméral est bien marqué. Le refroidissement nocturne est beaucoup plus important que dans le sol en début et en fin de cycle : les différences de température entre les 2 milieux sont nettement visibles à $9 \mathrm{~h}$ du matin, au moment des relevés (fig 6). Ceci n'a que peu d'importance sur la ramification des premiers entre-nceuds, qui, pour $E_{2}$, débute $35 \mathrm{j}$ après le semis, mais peut expliquer les résultats obtenus sur $E_{6}$ et $E_{7}$ où les premières secondaires apparaissent respectivement au bout de 74 et $85 \mathrm{j}$. Il est en effet admis (Cooper, 1973) que la ramification du maïs est fortement affectée par la température du sol.

Les observations effectuées in situ, à travers le plexiglas peuvent être utilisées en début de cycle pour déterminer les taux de ramification des premiers entre-nœuds en raison :

- des écarts relativement faibles entre les données du champ et celles des rhizotrons (primaires au cœur du monolithe de terre) sur $E_{2}, E_{3}, E_{4}$ et $E_{5}$;

- de l'importance des $C V$ sur les résultats.

\section{DISCUSSION ET CONCLUSION}

Les 3 points les plus importants mis en évidence par ce travail sont reportés ci-dessous :
- (i) Le manque de précision des estimations malgré un nombre de répétitions élevé. Nous observons in situ des CV d'environ $55 \%$ valeurs compatibles avec celles obtenues en rhizotrons par d'autres auteurs $(70 \%$ pour Taylor et Klepper, 1973 : étude sur maïs).

Les données recueillies au champ à partir des planches de fakir montrent qu'environ $30 \%$ de cette variabilité est inhérente au phénomène de ramification. Pour les autres comptages, viennent s'ajouter les conséquences des perturbations liées à la présence du plexiglas. Leur origine est soit mécanique (présence locale de fentes de retrait, Taylor et al, 1970; Huck et Taylor, 1982), soit physiologique. En effet, le plexiglas, qui accumule l'électricité statique attire les racines; pour le coton, leur nombre se voit multiplié par 1,5 (Taylor et Bohm, 1976; Voorhees, 1976). De plus, l'exposition des racines à la lumière pendant la durée des mesures limite leur vitesse de croissance (Bohm, 1979).

L'existence d'une densité de colonisation seuil au-delà de laquelle l'organogenèse serait fortement perturbée peut également être discutée. Le nombre de ramifications d'une portion de primaire serait alors inversement proportionnel à la densité racinaire de la fraction de sol au contact de cette primaire. L'effet dépressif du plexiglas observé en 1984 sur l'émission des secondaires corrobore cette hypothèse de régulation en feed back. Celle-ci a d'ailleurs déjà été envisagée par certains modélisateurs (Hackett et Rose, 1972; Hillel et al, 1976), mais n'a pas été démontrée expérimentalement. Elle est également étayée

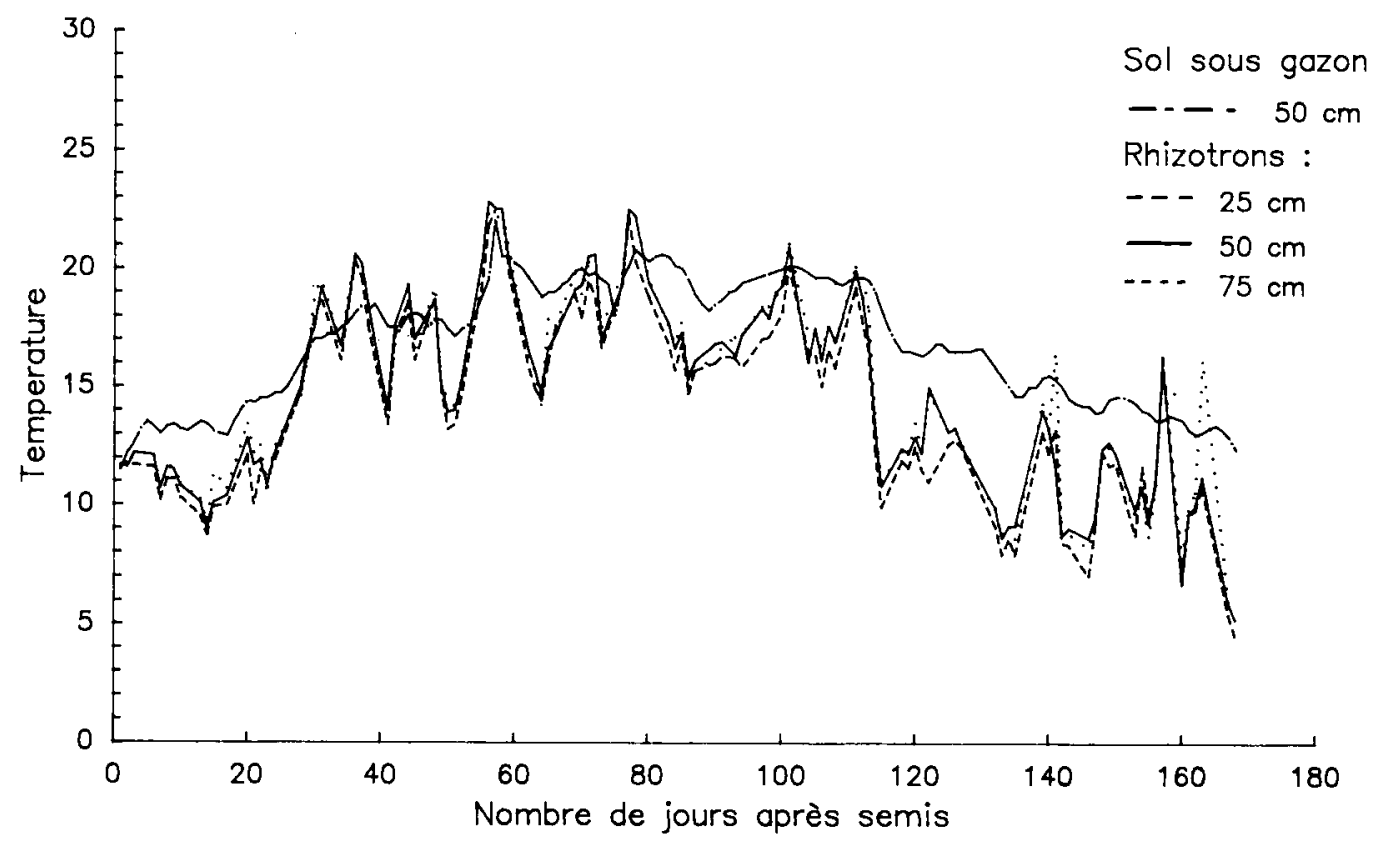

Fig 6. Température du sol, prise à 9 h du matin à différentes profondeurs. 
par le fait, que pour les céréales (riz, blé et orge) une augmentation du peuplement, donc de la densité, tend à réduire la longueur et le nombre des racines primaires et secondaires (Pavlychenko, 1937).

- (ii) La représentativité des observations effectuées à travers le plexiglas par rapport à ce qui se passe à l'intérieur du rhizotron. Ceci s'explique d'une part, par l'importance de la variabilité inhérente au processus de ramification, et, d'autre part, par le fait que les erreurs spécifiques aux mesures in situ, détaillées précédemment, restent les mêmes sur l'ensemble du cycle de culture;

- (iii) L'impossibilité, en fin de cycle, d'extrapoler ces données aux conditions du champ. Pour expliquer la divergence observée entre les $2 \mathrm{mi}-$ lieux de culture, il est possible d'incriminer d'autres facteurs caractéristiques de l'environnement pédoclimatique que la température dont l'importance a déjà été évaluée.

En rhizotron, chaque plante est isolée, donc non soumise aux compétitions existant au sein d'un peuplement ce qui, dans notre cas, a stimulé la synthèse de biomasse aérienne.

Le régime hydrique a été maintenu constant et non limitant dans les caissons, alors qu'au champ l'alimentation en eau est restée tributaire de la pluviométrie. Un léger stress a quelquefois été noté aux alentours de la floraison (Jordan, 1986). Ces conditions limites ont pu contribuer à stimuler la ramification sur les derniers entrenœuds (Weaver, 1926; Lambers et Posthumus, 1980).

L'adéquation des données du rhizotron aux conditions habituelles de culture n'est donc pas immédiate. En effet, elle suppose :

- soit, une dérive constante au cours du temps entre les différentes méthodes de mesure. Ce n'est pas systématique et ce point devra être vérifié pour chaque expérimentation. Dans le cadre de notre étude, par exemple, les résultats des observations effectuées, d'une part à travers le plexiglas et d'autre part à l'aide des planches de fakir ne sont liés par le même coefficient de proportionnalité que pour les entre-nœuds de rang inférieurs à 5. Taylor et Klepper (1973), Voorhees (1976) montrent au contraire que, pour les densités racinaires, les résultats du caisson (évaluation à travers la paroi) sont inférieurs de $6 \%$ a ceux du champ et que les cinétiques d'évolution au cours du cycle sont comparables dans les 2 cas. Ces données ont été obtenues à Auburn (Alabama) sur un dispositif enterré particulière- ment bien isolé du point de vue thermique (Taylor, 1969). II est donc légitime, compte tenu des remarques précédentes, de penser que ces résultats sont plus représentatifs des conditions habituelles de culture que les nôtres, particulièrement en fin de cycle;

- soit la modélisation de l'effet induit par chacune des composantes du milieu (température, humidité). Ce type de travail à déjà été effectué, en conditions contrôlées, pour la vitesse de croissance de la racine séminale (Wang, 1983) d'une jeune plantule de maïs. Cependant le passage à l'échelle du champ n'a pas été possible pour 2 raisons : la nécessité de prendre en compte l'effet des interactions entre les différents facteurs et la difficulté à travailler sur un végétal adulte dont les différents réseaux racinaires sont soumis à des régulations de croissance (trophique ou hormonale) dont les modalités sont encore très mal connues.

Cependant, si l'on émet l'hypothèse que les écarts observés entre le champ et les caissons pour l'horizon $0 / 35 \mathrm{~cm}$ ne sont pas modifiés de façon significative par la profondeur, les rhizotrons restent l'outil le mieux adapté pour l'étude de la croissance et du développement du système racinaire à l'échelle de la plante entière. En effet, certains paramètres (vitesses de croissance racinaires, rythmes d'émission des racines d'ordre 2 et 3 ), ne peuvent pas être évalués avec précision en conditions habituelles de culture. Les techniques d'endoscopie (Maertens et Clauzel, 1982; Manantsoa, 1984) développées récemment, et utilisées principalement pour évaluer la densité de colonisation d'un sol, ne donnent que des résultats fragmentaires en ce qui concerne la structure racinaire. II est, par exemple, difficile de déterminer le rang d'insertion des primaires dont dépendent pourtant plusieurs composantes de l'architecture racinaire (Jordan, 1986). De plus, chaque racine ne peut être suivie que pendant une période relativement courte, ce qui pose des problèmes d'échantillonnage. Le développement de ces techniques est cependant indispensable pour évaluer la représentativité, à l'échelle de la parcelle, des données obtenues en caisson. Cette validation est en effet indispensable pour toute étude quantitative en rhizotron.

\section{RÉFÉRENCES}

Bohm W (1979) Methods for studying root systems. Ecological Studies 33, Springer-Verlag, Berlin, 188 p 
Cooper AJ (1973) Root temperature and root growth. Commonw Agric Bureaux Slough, $73 \mathrm{p}$

De Roo HC (1957) Root growth in Connecticut tobacco soils. Conn Agric Exp Stn, New Haven, CT, 608, $1-36$

Girardin P, Jordan MO, Picard D, Trendel R (1986) Harmonisation des notations concernant la description morphologique d'un pied de maïs (Zea mays L). Agronomie 6, 873-875

Grimes DW, Miller RJ, Willey PL (1975) Cotton and corn root development in two field soils of different strength characteristics. Agron J 67, 519-523

Hackett C, Rose DA (1972) A model of the extension and branching of a seminal root of barley and its use in studying relations between root dimensions. I. The model. Aust J Biol Sci 25, 669-679

Hillel D, Talpaz H, Van Keulen H (1976) A macroscopic model of water uptake by a non uniform root system and of water and of soil movement in the soil profile. Soil Sci 121, 242-255

Huck MG, Taylor HM (1982) The rhizotron as a tool for root research. Adv Agron 35, 1-35

Jordan MO (1986) Mise en place du système racinaire du maïs. Définition et caractérisation au champ et en conditions contrôlées des paramètres descriptifs de la structure racinaire. Construction de l'organigramme d'un modèle cinétique. Thèse (Doctorat d'université), Université Louis Pasteur, Strasbourg, $177 \mathrm{p}$

Jordan MO, Picard D, Trendel R (1992) Ramification secondaire du maïs (Zea mays $L$ ). Données de structure et de cinétique. Agronomie 12 (1), 15-30

Lambers $H$, Posthumus $F(1980)$ The effect of light intensity and relative humidity on growth rate and root respiration of Plantago lanceolata and Zea mays. J Exp Bot 31, 1621-1630

Maertens C, Clauzel Y (1982) Premières observations sur l'utilisation de l'endoscope dans l'étude de l'enracinement des plantes cultivées (Sorghum vulgare et Lolium multiflorum). Agronomie 2, 677-680
Manantsoa V (1984) Conception et réalisation d'un endoscope automatique ou rhizoscope pour l'étude de la dynamique racinaire des plantes in situ. Thèse de $3^{e}$ cycle, Université de Haute Alsace, Mulhouse, $171 \mathrm{p}$

Nelson WW, Allmaras RR (1969) An improved method for excavating and describing roots. Agron $J 61$, 751-755

Pavlychenko TK (1937) Quantitative study of the entire root systems of weed and crop plants under field conditions. Ecology 19, 62-79

Peterson CM, Klepper B, Pumphrey FV, Rickman RV (1984) Restricting rooting decreases tillering and growth of winter wheat. Agron J 76, 861-864

Picard D, Jordan MO, Trendel R (1985) Rythme d'apparition des racines primaires du maïs (Zea mays L). 1. Étude détaillée pour une variété en un lieu donné. Agronomie 5, 667-676

Raper CD, Barber SA (1970) Rooting system of soybeans. I. Differences in root morphology among varieties. Agron J 62, 581-584

Taylor HM (1969) The rhizotron at Auburn, Albama. A plant root observation laboratory. Circulaire 171, Agric Exp Stn, Auburn, Alabama, $10 \mathrm{p}$

Taylor HM, Bohm W (1976) Use of acrylic plastic as rhizotron windows. Agron J 68, 693-694

Taylor HM, Huck MG, Klepper B, Lund ZF (1970) Measurement of soil grown roots in a rhizotron. Agron J 62, 807-809

Taylor HM, Klepper B (1973) Rooting density and water extraction patterns for corn (Zea mays $\mathrm{L}$ ). Agron J 65, 965-968

Voorhees WB (1976) Root elongation along a soilplastic interface. Agron J 68, 143

Wang $J$ (1983) Growth simulation of the corn radicle root for various soil physical conditions. PhD Pennsylvania State Univ, 194 p

Weaver JE (1926) Root habits of maize. In: Root development of field crops. Mc Graw-Hill Book Co, NY, 180-191 